\title{
A Computational Treatment of Sentence-Final 'then'
}

\author{
Sheila Glasbey \\ Centre for Cognitive Science \\ Edinburgh University \\ 2 Buccleuch Place \\ Edinburgh EH8 9LW \\ UK
}

\begin{abstract}
We describe a computational system which parses discourses consisting of sequences of simple sentences. These contain a range of temporal constructions, including time adverbials, progressive aspect and various aspectual classes. In particular, the grammar generates the required readings, according to the theoretical analysis of (Glasbey, forthcoming), for sentence-final 'then'.
\end{abstract}

\section{Sentence-final 'then'}

It is possible to follow:

(1a) Emily climbed Ben Nevis in July. with

(1b) Fiona climbed Snowdon then.

This is interpreted to mean that each climb took place at some time within the July in question. Notice, however, that if we remove 'in July' from (1a) to give:

(1c) Emily climbed Ben Nevis.

the sequence $(1 c, 1 b)$ becomes harder to interpret and sounds rather odd. ${ }^{1}$ The difference is, of course, that we have removed 'in July' and there is no longer an explicit reference to a "time". We will call such an explicitly mentioned time an explicit temporal referent (ETR). Thus, sentence-final 'then' appears,

\footnotetext{
${ }^{1}$ We are not concerned here with the rather marginal reading, available to some speakers, where what is conveyed by $(1 c, 1 b)$ is that Fiona's climb follows Emily's. This corresponds to the "updating" reading normally associated with sentence-initial 'then'.
}

on the basis of this and other examples, to require explicit mention of a time. Being able to infer a time from the description of an event is clearly not enough. We would expect to be able to infer readily from (1c) that there was a time at which Emily's climb took place. However, it appears that we cannot use sentence-final 'then' here to refer back to such an inferred time.

In order to make sense of the sequence $(1 c, 1 b)$ without the ETR, it seems we have to be able to see the two events as connected in some way. Consider:

(1c) Emily climbed Ben Nevis.

(1d) She achieved her ambition then.

which sounds fine, and:

(2a) The children went to Wales.

(2b) Fiona climbed Snowdon then.

which is also perfectly acceptable. Note that in both these cases the second event is readily seen as connected to the first-by the kind of discourse relation that has often been called elaboration. ${ }^{2}$

Now consider:

(3a) John went to France.

(3b) Bill Clinton became president then.

This sequence sounds odd, presumably because it is difficult to see any connection between the events described in (3a) and (3b). Consider also:

(4a) John took the children to Aviemore.

(4b) Mary wrote her paper then.

which sounds odd if we do not know who Mary is,

\footnotetext{
${ }^{2}$ See, for example, (Mann and Thompson, 1987).
} 
but sounds fine if we are told that John and Mary are the parents and John took the children off to Aviemore to give Mary peace and quiet to write her paper. In other words, the sequence is acceptable if we can envisage a connection between the events.

On the basis of these examples, it appears that sentence-final 'then' either requires an ETR, or there must be some kind of connection, such as an elaboration relation, between the two events.

The picture is still incomplete, however. The examples considered so far have been accomplishments or achievements. ${ }^{3}$ If the second sentence of the sequence is a lexical stative or a progressive ${ }^{4}$, sentence-final 'then' becomes acceptable even when the first sentence contains no ETR and there is no obvious connection between the eventualities. ${ }^{5}$

For example, $(1 c, 1 \mathrm{e})$ and $(1 c, 1 \mathrm{f})$ are both perfectly acceptable.

(1c) Emily climbed Ben Nevis.

(1e) Fiona was a girl then.

(1f) Fiona was climbing Snowdon then.

A detailed analysis of such sequences, which was carried out in (Glasbey, forthcoming) and (Glasbey, ms1), reveals the importance of the notion of discourse backgrounding. Provided that the sequence can be interpreted in such a way that the second eventuality is presented as backgrounded with respect to the first, sentence-final 'then' is acceptable and the sequence $(1 \mathrm{c}, 1 \mathrm{e})$, for example, conveys that Emily's climb is temporally included in the state of Fiona's being a girl. A similar notion in the literature is that of the temporal overlap often conveyed when a stative (or progressive) follows a non-stative; see, for example, (Hinrichs, 1986). We will show in Section 2 how the notion of discourse backgrounding can be formalized in our theoretical framework.

We have seen, too, that sentence-final 'then', in the absence of an ETR, is acceptable in cases where the second eventuality can be seen as an elaboration of the first. This means that we have so far identified three uses of sentence-final 'then':

\section{The ETR use.}

2. The elaboration use.

3. The background use.

It would simplify matters if we could group (2) and (3) together-perhaps by saying that backgrounding is another way of expressing a connection between two events.

In our formal analysis, to be described shortly, which uses the situation theory/discourse representation theory (ST/DRT) framework of (Barwise and

\footnotetext{
${ }^{3}$ We use the terminology of (Vendler, 1967).

${ }^{4} \mathrm{Or}$ an iterative state or habitual state, using the terminology of (Moens, 1987).

${ }^{5}$ We use this term to include events and states, as in (Bach, 1986).
}

Cooper, forthcoming), we model eventualities as situations. We express the connection between eventualities by means of the situation-theoretic relation part-of (or $\unlhd$ ), from (Barwise, 1989). Part-of is a relation ${ }^{6}$ which holds between situations. In order for sentence-final 'then' to be acceptable in the absence of an ETR, the second eventuality must be part-of the first. This intuitively covers the elaboration case, in that it makes sense to think, for example, of Fiona's climbing Snowdon as being part of the children's trip to Wales in $(2 a, 2 b)$. But how does it work in the backgrounding case? We will explain in Section 2, when we have introduced some notation, how the part-of analysis can be used to cover this case too.

If we take the part-of analysis to cover both the backgrounding and elaboration cases, we can now say that there are two distinct uses of sentence-final 'then'. The first involves reference back to a previously introduced ETR and is only possible if such an explicit referent is present. The second does not refer to an explicit time, but rather conveys that the second eventuality is part-of the first. This may be the case if the second sentence is stative or progressive. Of course, progressives have often been analysed as stative in the literature (for example, by Vlach (1981)). Part of the motivation given for the progressive-as-stative analysis concerns facts about temporal overlap and updating. We prefer to say that an event described in the progressive is interpreted as backgrounded with respect to a previous (nonprogressive) event in the discourse. ${ }^{7}$ We thus keep separate the notions of stativity and backgrounding, which enables us to explore the relationship between the two concepts. ${ }^{8}$ We adopt Smith's two-component theory of aspect (Smith, 1991) and regard progressive aspect as conveying an internal perspective or viewpoint on the described event.

The part-of relation between eventualities may also hold if the second eventuality can be read as an elaboration of the first. Of course, world knowledge will often be required to decide this. Part-of is therefore a relation between two eventualities which covers both the background and the elaboration discourse relations.

Thus we see that sentence-final 'then' can, if conditions are right, give rise to two readings. This is shown in sequences where the conditions for ETR 'then' and those for part-of 'then' are both fulfilled. For example:

\footnotetext{
${ }^{6}$ In our formal treatment we will in fact treat $\unlhd$ as a type, but this is a technical detail. We will continue to refer to the ' $₫$ relation' rather than the ' $\triangle$ type', as the former conveys a clearer meaning.

${ }^{7}$ Actually there are cases where a progressive does not convey backgrounding, but we will not discuss them here. They involve 'at the same time' and are discussed in (Glasbey, ms1).

${ }^{8}$ See (Glasbey, ms1, Glasbey, ms2) for details.
} 
(1a) Emily climbed Ben Nevis in July.

(1f) Fiona was climbing Snowdon then.

(1a,1f) can either mean that Fiona's climb took place in July, or that it temporally included Emily's climb. World knowledge or context may sometimes favour one reading or the other.

This analysis of sentence-final 'then' has important consequences for theories of temporal reference. It shows that, whatever theoretical framework is employed, it is necessary to distinguish in some way between temporal discourse referents which are introduced into the discourse via explicit mention of a time, and those which are introduced via the inference of a time from the mention of an event or state. We explain below a means of making this distinction in an ST/DRT framework, and describe a computational implementation which embodies the distinction. ${ }^{9}$

\section{Grammar and Implementation}

The fragment contains sequences of sentences of a type similar to the ones given in Section 1. It includes sentence-final 'then', together with other temporal adverbials such as for-adverbials, frame adverbials (e.g, 'in July') and completive in-adverbials (e.g., 'in two hours'). Sentence-initial 'then' and sentencefinal 'at the time' and 'at the same time' are also included, although we do not discuss their analysis here. There is a range of verbs, transitive and intransitive, with various aspectual characteristics, and a range of noun types including count nouns, mass nouns, bare plurals, definite and indefinite NPs. Progressives are also included. We are thus concerned not merely with the analysis of 'then' but with matters of aspectual composition/modification and the distribution of temporal adverbials. Space does not permit us to describe the full system in detail. We will concentrate here on those parts of it that are particularly relevant to the analysis of 'then'.

As the system is concerned with temporal matters, we have not built into it a treatment of pronominal anaphora. However, it is designed in such a way, as will shortly become clear, that it could be extended without undue difficulty to include pronoun anaphora, using a treatment based on that in (Johnson and Klein, 1986).

The system parses sequences of sentences and produces representations for the required readings for

\footnotetext{
${ }^{9}$ We discuss in (Glasbey, msl) how 'at the time' behaves similarly to the par' $t$-of use of 'then' (but conveys only backgrounding and not elaboration), while 'at the same time' appears to be acceptable in cases where the second eventuality is not a part of the first, i.e., where it can be seen as forming a distinct or separate event. These are also included in the implemented grammar, but their treatment is not described here.
}

sentence-final 'then'. It is based on a situationtheoretic grammar developed in (Cooper, 1991) and its computational implementation ProSit (Cooper, msl). ProSit is a definite clause grammar (DCG) with features. It parses single sentences and constructs syntactic and semantic representations expressed in situation-theoretic terms. We have extended it firstly to deal with sentences containing a range of tense and aspect constructions which were not present in Cooper's original fragment, and secondly to allow the processing of discourse. To enable us to do the former, we have built aspectual composition into the grammar using a theoretical approach based upon (Krifka, 1991) and described below. In order to process discourse, we have employed the technique known as 'threading', used by Johnson and Klein (1986), whereby discourse referents are carried from left to right among the constituents of a sentence, and from one sentence to the next.

\section{Extended Kamp Notation}

The grammar is expressed in a combined DRT/situation theoretic formalism, employing the Extended Kamp Notation (EKN) developed in (Barwise and Cooper, forthcoming). These authors use a box notation for situation-theoretic objects such as infons, situations and propositions, based upon the graphical notation of DRT (Kamp and Reyle, forthcoming). However, in EKN the boxes directly represent semantic objects, in contrast to DRT where the discourse representation structures (DRSs) are expressions of a language which require interpretation in a model. Nevertheless, EKN boxes look rather like DRSs. One important difference, however, is that EKN boxes may contain situations.

In situation theory, infons (which can be thought of as items of information or "possible facts") are supported by situations, which are parts of the world as individuated by agents. An infon consists of a relation ${ }^{10}$ with its argument roles filled by objects which may be individuals, parameters or other situation-theoretic objects. Propositions in EKN include objects of the form:

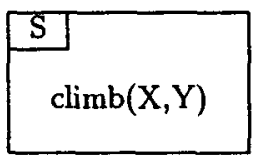

which is the proposition that a situation $\mathbf{S}$ supports an infon $\operatorname{climb}(X, Y) .{ }^{11}$ Situation-theoretic objects may have restrictions imposed on them. A proposition with restrictions is shown in Figure 1.

The box in Figure 1 denotes an object only if the restrictions are true, i.e., in the above case, if $\mathrm{X}$ is

\footnotetext{
${ }^{10}$ Relations are primitives in situation theory.

${ }^{11} \mathrm{~S}, \mathrm{X}$ and $\mathrm{Y}$ are parameters, denoted by capital letters in situation theory. A parameter is a partially-specified object.
} 


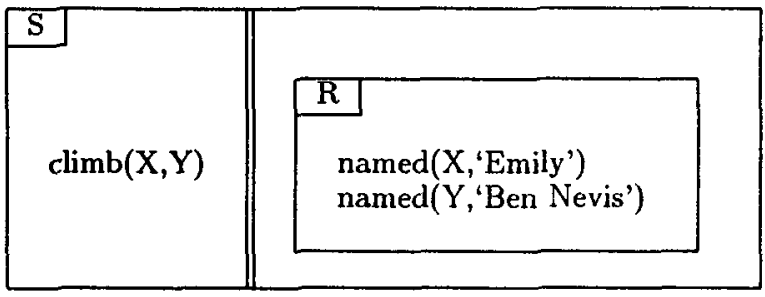

Figure 1: An EKN restricted proposition.

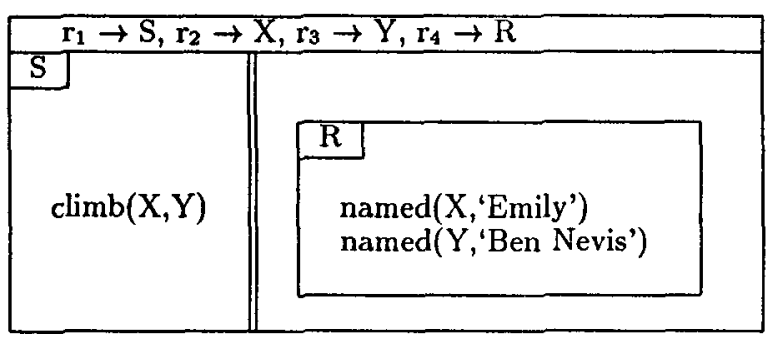

Figure 2: An EKN proposition abstract or 'type'.

anchored to an individual named 'Emily' and $Y$ to an individual named 'Ben Nevis'. $R$ is the resource situation supporting information about the naming of individuals. ${ }^{22} \mathrm{~A}$ proposition containing parameters is known as a parametric proposition. It is possible to abstract (simultaneously) over one or more parameters of a parametric proposition to give a type of the form shown in Figure 2.

Once a parameter has been abstracted over, it effectively "disappears" and is no longer present in the type. What remains is the "role" corresponding to the abstracted parameter. These roles may be indexed however we choose (for example, by the natural numbers, by $r_{1}$ to $r_{n}$ as above, or by utterance situations as in (Cooper, 1991)).

Cooper (ms2), in the development of situationtheoretic DRT (STDRT), sees a DRS as equivalent to the situation-theoretic type obtained by abstracting over the parameters of a proposition. The roles of such a type are equivalent to DRT discourse referents, and the infons correspond to the conditions of the "main" situation. ${ }^{13}$

\section{Processing of Sentences}

The system parses both individual sentences and sequences of sentences forming a discourse. For a sentence such as:

(1c) Emily climbed Ben Nevis.

it produces a syntactic parse tree, together witlr a semantic representation in the form of a DRS/type as shown in Figure 3. The DRS/type is shown in slightly simplified form here. It will also contain in-

\footnotetext{
${ }^{12}$ See (Cooper, forthcoming) for further explanation.

${ }^{13}$ Of course there are no precise DRT equivalents of the situation and the restrictions.
}

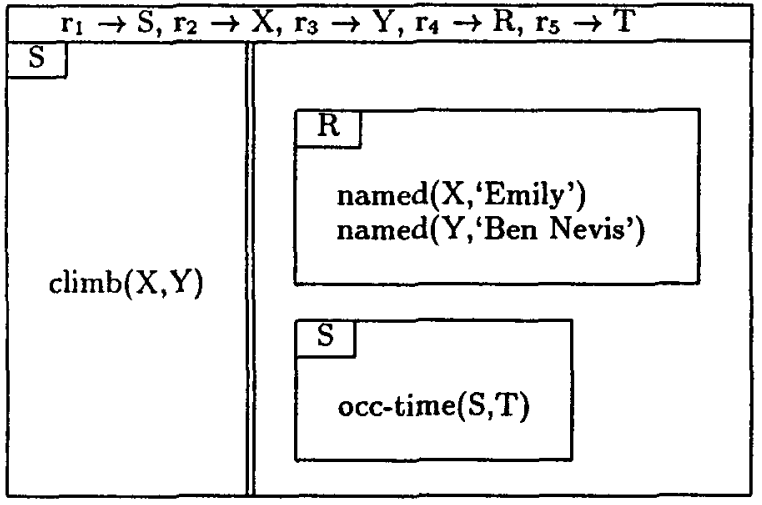

Figure 3: DRS/type for (1c).

formation about aspectual class etc., as discussed below.

Parsing of an individual sentence takes place in a top-down ${ }^{14}$, left-to-right manner, causing a DRS/type like the one in Figure 3 to be gradually built up. The lexical entry for a verb introduces a "skeletal" (partially instantiated) type, and further information is added to this by the remaining constituents as parsing proceeds.

Although there is no explicit mention of a "time" in (1c), the representation for this sentence (Figure 3) contains a parameter $\mathrm{T}$ corresponding to what we call the the "occurrence time" of the eventuality. This is the total temporal extent of the eventuality. Although inclusion of the occurrence time is not strictly necessary in the representation for a single sentence with no ETR, it will be needed when we come to process discourse. We will see shortly that stative verbs do not introduce occurrence-times into the representations, whereas non-stative ones do, unless they are presented with progressive aspect.

Now compare the representation produced for the sentence:

\section{(1a) Emily climbed Ben Nevis in July.}

In this case, the system produces the DRS/type shown in Figure 4.

Here we have a second temporal parameter $\mathbf{T}^{\prime}$, corresponding to to the explicit temporal referent 'July'. Note that the role corresponding to this parameter is indexed by ' $p r$ '. This indicates that this time referent, unlike the one corresponding to $\mathrm{T}$, is phonologically realised in the utterance. This distinction will be important when we come to process 'then'. Here we are exploiting the possibility afforded by situation theory of being able to include information about the utterance in our semantic representations. ${ }^{15}$

\footnotetext{
${ }^{14}$ However, top-down processing is not essential to the grammar, and a left-corner parser or chart parser could be used instead.

${ }^{15}$ We have not taken the trouble here to mark non-
} 


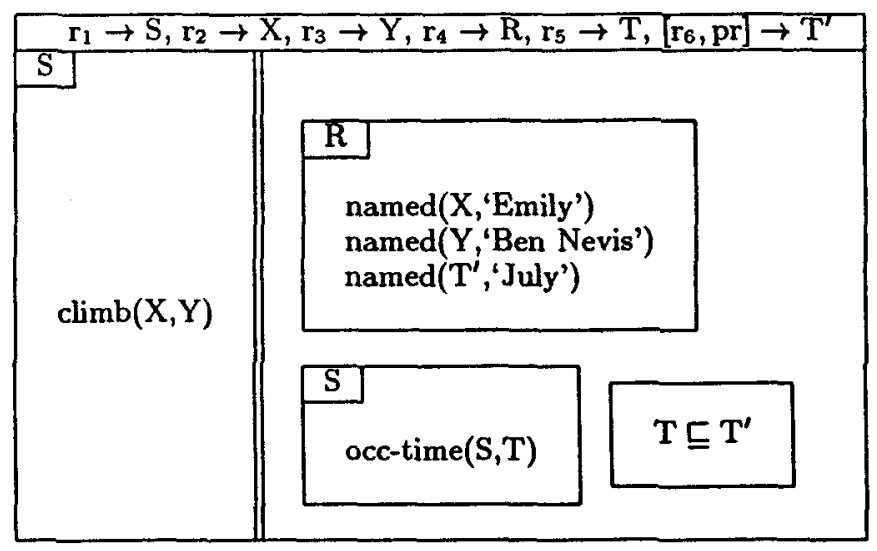

Figure 4: DRS/type for (1a),

The box:

$$
\mathbf{T} \subseteq \mathbf{T}^{\prime}
$$

is another kind of EKN proposition-one that does not involve a situation. It expresses the information that $T$ and $T^{\prime}$ are of type $\subseteq$, where this is a type of two times such that the second includes or equals the first.

\section{Processing of Discourse}

Now let us consider the semantic representation for a discourse. This consists of a proposition which is the conjunction of the propositions introduced by the individual sentences. Abstraction is carried out over the conjoined proposition as a whole, giving a list of discourse referents/roles for the discourse processed up to a given point.

Thus for $(1 \mathrm{a}, 1 \mathrm{~g})$ :

(1a) Emily climbed Ben Nevis in July.

(1g) Fiona climbed Snowdon.

we get the representation shown in Figure $5 .{ }^{16}$

Now let us consider the processing of discourse sequences containing sentence-final 'then'. Consider $(1 \mathrm{a}, 1 \mathrm{~b})$ :

(1a) Emily climbed Ben Nevis in July.

(1b) Fiona climbed Snowdon then.

The system parses (1a), followed by (1b) as far as 'then'. At this point in processing, the representation built so far is that of Figure 5. The processing

temporal discourse referents as phonologically realised, as this is not relevant to the analysis of 'then'-but it could of course be done.

${ }^{16}$ The representation for $(1 \mathrm{a}, 1 \mathrm{~g})$ will also contain information about possible discourse relations between the two eventualities. We do not describe this feature of the system here except where it is relevant to 'then'. of 'then' causes the rules for ETR 'then' and partof 'then' to be invoked in turn. The rule for ETR 'then' causes the system to "look for" a temporal referent indexed ' $\mathrm{pr}$ ' in the list of discourse referents introduced by the processing of the discourse up to this point. This list of discourse referents is threaded from one sentence to the next (and from NP to VP within a sentence). In fact, what is threaded is not just the discourse referents but the overall DRS/type from the processing of the discourse up to this point. The threading is achieved at discourse level by means of the top-level rule of the grammar:

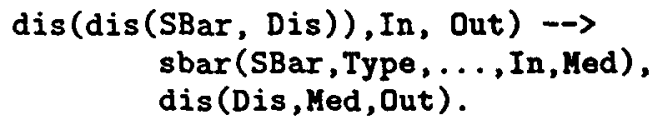

The first argument to the predicate 'dis' is responsible for building the tree structure associated with the parse. The second and third arguments, the Prolog variables 'In' and 'Out', enable threading of discourse referents from the sentence just parsed to the remaining discourse. The input 'In' to the processing of sbar consists of the overall DRS/type built up from processing the discourse up to this point. This includes a list of discourse referents generated so far. The grammar rules at sbar level and below cause the overall DRS/type to be updated to give a new type 'Med', which is the input DRS/type to the processing of the remainder of the discourse. The 'Type' argument of sbar is the DRS/type obtained from parsing that individual sentence. The other arguments to sbar are not relevant to this discussion and have thus been omitted.

Thus, at a given point in processing of discourse, the system can look for a temporal referent indexed 'pr'. Looking at Figure 5, we see that an appropriate temporal referent indexed ' $\mathrm{pr}$ ' is present. The rule for ETR 'then' therefore succeeds, and a proposition is introduced to the effect that $T_{2}$ is temporally included in $\mathrm{T}^{\prime}$, i.e. 


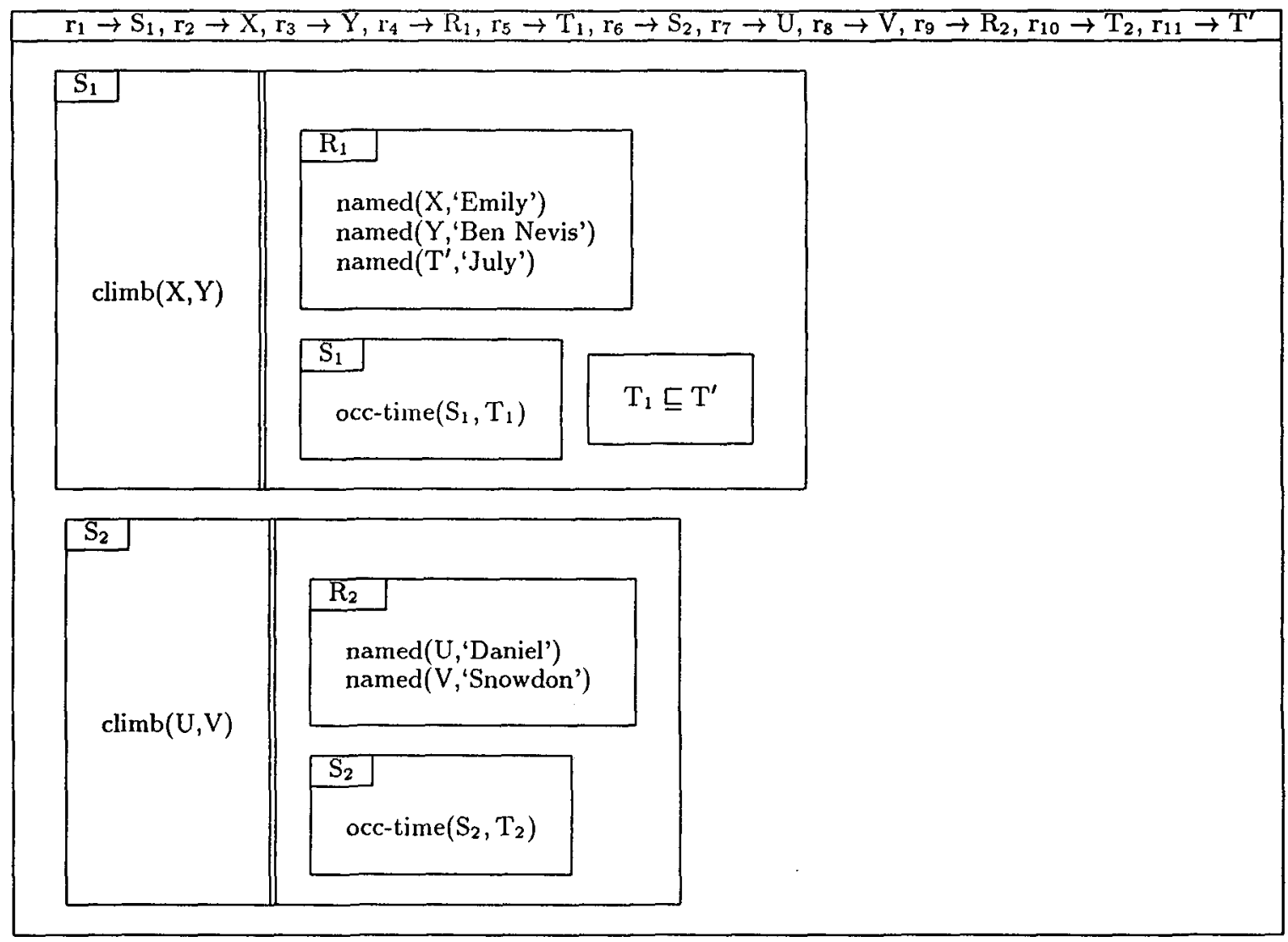

Figure 5: Slightly simplified representation for $(1 \mathrm{a}, 1 \mathrm{~g})$ and for $(\mathrm{la}, 1 \mathrm{~b})$ at the point of processing 'then'. 


$$
\mathrm{T}_{2} \sqsubseteq \mathrm{T}^{\prime}
$$

This proposition is added to the restrictions of the lower box of Figure 5, to give the completed representation for $(1 \mathrm{a}, 1 \mathrm{~b})$, which is not shown here for reasons of space. If there had been no such temporal referent marked 'pr' present, the rule for ETR 'then' would have failed.

Now consider the part-of reading for 'then'. We saw earlier that this requires an appropriate discourse relation between the two described eventualities - one of either backgrounding or elaboration. Testing for whether an elaboration relation is possible requires world knowledge, and we have not attempted to build any of this into the system, although there appears to be no reason why this could not be done. The system in its present form therefore checks only for the background instance of the part-of relation.

Backgrounding is possible if the second eventuality is either a state or if it is presented with prom gressive viewpoint. This means that, in order to test for backgrounding, the representations for individual sentences must contain information about the aspectual properties of the described eventualitiesfor example, whether an eventuality is a state or a non-state (event), and whether it is presented with simple aspect (external viewpoint) or progressive aspect (internal viewpoint). It is widely known that the aspectual properties of a described eventuality depend on certain properties of the verb ${ }^{17}$ and also on other elements such as the referents of NP arguments. For example, the event described by:

(5) Daniel climbed a mountain.

is a Vendler accomplishment. Alternatively, we may characterise it in Krifka's terms as having the property $+Q$ (quantized) or -CUM (non-cumulative), which are equivalent to the lack of a natural endpoint or culmination. However, the event described by:

(6) Daniel climbed mountains.

is a Vendler activity, and in Krifka's terms has the property $-Q /+C U M$. Here we see what Krifka describes as a "mapping" from the properties of the NP object $^{18}$ to the properties of the event. The referent of 'a mountain' is $+Q$, and so is the event of (5). The referent of 'mountains' is $-Q$, and so is the event of (6). Such mapping from the properties of the object to the properties of the event only occurs for certain verbs, however-those where what Krifka calls the

\footnotetext{
${ }^{17}$ E.g. 'basic aspectual type' in Moens' terms (Moens, 1987) and semantic features in both Verkuyl's (1989) and Krifka's (1991) accounts.

${ }^{18}$ More strictly the "patient", as it is thematic roles and not grammatical roles that are important here.
}

"thematic relation" between the object and the event has an appropriate property. One such property that enables this mapping is what he calls gradual patient. In such cases, there is an intuitive relationship between the "progress" of the object and the progress of the event. For example, in an eating event, the object is gradually consumed, and in a writing event, the object is gradually created. Both 'eat' and 'write', as well as 'climb' thus have thematic relations with the property gradual patient. Driving events do not, on the other hand, exhibit this correspondence between the progress of the event and the progress of the object. Thus the thematic relation between object and event for 'drive' does not have the gradual patient property, which explains why:

(7) John drove the car.

is +CUM/-Q even though 'the car' is $-\mathrm{CUM} /+\mathrm{Q} .^{19}$

In our EKN account we encode Krifka's properties of thematic relations as types of situations and individuals. For example, the lexical entry for 'climb' includes the following information:

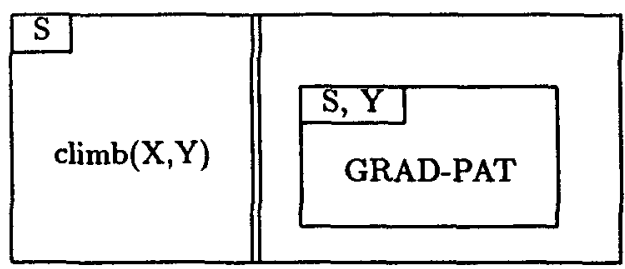

The grammar rules then make reference to this information. For example, the rule:

$$
\operatorname{vbar}(\ldots) \rightarrow r(\ldots), n p(\ldots)
$$

contains a procedure which evaluates the $\mathrm{Q}$-value of the predicate (vbar) according to the following algorithm:

If: The thematic relation between $\mathrm{S}$ and $\mathrm{Y}$ is of type GRAD-PAT

Then: Set the Q-value of the predicate (vbar) to be the same as that of $Y$

Otherwise: Set the $Q$-value of the predicate to $-Q$.

The Q-value of the agent ${ }^{20}$ also affects that of the described eventuality. For example, the eventuality described by:

(8) Emily climbed the mountain.

is $+Q$, whereas that described by:

(9) People climbed the mountain.

is $-Q$. In (9), the $-Q$ value of the agent is transferred to the event. In order to deal with such examples, the rule

$s(\ldots) \rightarrow n p(\ldots), \operatorname{vp}(\ldots)$

\footnotetext{
${ }^{19} \mathrm{~A}$ well-known test for the property $+\mathrm{CUM} /-\mathrm{Q}$ of predicates is the ability to combine with a for-adverbial.

${ }^{20}$ Corresponding to the grammatical subject in these active sentences.
} 


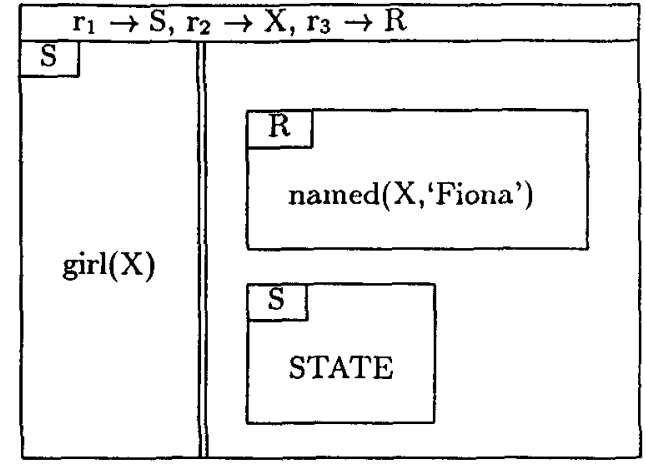

Figure 6: Representation for (1e) at the point of processing 'then'.

contains a similar algorithm to the one in the vbar rule.

Thus the representation constructed by parsing a sentence includes information about the aspectual properties of the described eventuality. These include the features $+/-$ STATE and $+/-Q$ as already described, together with $+/-$ PROG depending on whether or not progressive aspect is present, and $+/$-PUNCT which distinguishes punctual and nonpunctual events (corresponding to the difference between achievements and accomplishments).

Let us now consider the representation from the processing of:

(1e) Fiona was a girl then.

up to the point where 'then' is reached. This is given (in slightly simplified form) in Figure 6.

Now suppose we are processing (1c,1e):

(1c) Emily climbed Ben Nevis.

(1e) Fiona was a girl then.

The rule for part-of 'then' requires that the second eventuality is either a state or it is described with progressive viewpoint. The former is true in this case, so the conditions for part-of 'then' are satisfied. The representation obtained for $(1 c, 1 e)$ is shown in Figure 7 .

\section{The semantics of 'part-of'}

What exactly does it mean for the part-of $(\unlhd)$ relation to hold between two eventualities? The idea is that if $S_{2} \unlhd S_{1}$, then any infon which is supported by $S_{2}$ is also supported by $S_{1}$. In other words, $S_{2}$ adds further information to $S_{1}$, causing it to be more fully specified. Here we exploit the partiality of situation theory. Situations may be only partially specified: if we say that $S_{1}$ supports $\sigma$, this does not tell us anything about what other information $S_{1}$ does or does not support. It is thus possible for a later utterance to add further information about $S_{1}$ and thereby specify it more fully. If the first utterance tells us that $S_{1}$ supports the infon $\sigma$, and the second tells us that $S_{2}$ supports the infon $r$ and also that $S_{2} \unlhd S_{1}$, then we know that $S_{1}$ supports both $\sigma$ and $\tau$. This is straightforward enough for the elaboration case. We need to consider carefully what it means in a backgrounding case such as $(1 \mathrm{a}, 1 \mathrm{e})$.

According to our theoretical analysis, if an eventuality is backgrounded then it does not introduce an occurrence-time of its own. Instead, the backgrounded eventuality is of the same duration as that of the preceding event-it "takes on" the time of that event. $^{21}$ Thus, in the representation of $(1 c, 1 \mathrm{e})$ in Figure 7 , the backgrounded $S_{2}$ has the same temporal extent as the event $S_{1}$. This amounts to claiming that (le) describes only the part of the state that coincides with the preceding event. Of course we know that the state of Fiona's being a girl began before and continues after Emily's climb-there is a relationship of temporal inclusion between the "total duration" of the state and the event. But we are saying that those parts of the state that are before and after the event are not described but are inferred from our world knowledge about the duration of such states.

Stative verbs are "natural backgrounders" in that they describe eventualities without making reference to the beginning and end points of the eventuality. They naturally describe a situation which can readily be seen as a temporal "slice" of a more prolonged situation. For this reason, in the lexical entries for stative verbs in our grammar, there is no mention of the occurrence-time of the state. Progressives usually behave in a similar way. When an event described with progressive viewpoint follows one with simple (perfective) viewpoint, the relation between them is normally one of backgrounding. The effect of progressive viewpoint is to present the event from an internal perspective. An event described with internal perspective is no longer temporally bounded-it does not have an occurrence-time of its own. Instead, its duration is that of the preceding event, just as in the stative case.

If we define two instances of the part-of relation:

- $\unlhd_{b g}$ for the backgrounding case

- $\unlhd_{e l}$ for the elaboration case

we can thus say:

$$
\mathrm{S}_{2} \unlhd_{\text {bg }} \mathrm{S}_{1} \longrightarrow \mathrm{T}_{2}=\mathrm{T}_{1}
$$

where $T_{1}, T_{2}$ are the temporal durations of $S_{1}$ and $S_{2}$ respectively. And:

$$
\mathrm{S}_{2} \unlhd_{\mathrm{el}} \mathrm{S}_{1} \longrightarrow \mathrm{T}_{2} \sqsubseteq \mathrm{T}_{1}
$$

Thus, for the general $\unlhd$ relation:

$$
\mathrm{S}_{2} \unlhd \mathrm{S}_{1} \longrightarrow \mathrm{T}_{2} \sqsubseteq \mathrm{T}_{1}
$$

\footnotetext{
${ }^{21}$ Evidence for this comes from an analysis of 'at the time' and 'at the same time'. See (Glasbey, ms2) for details.
} 


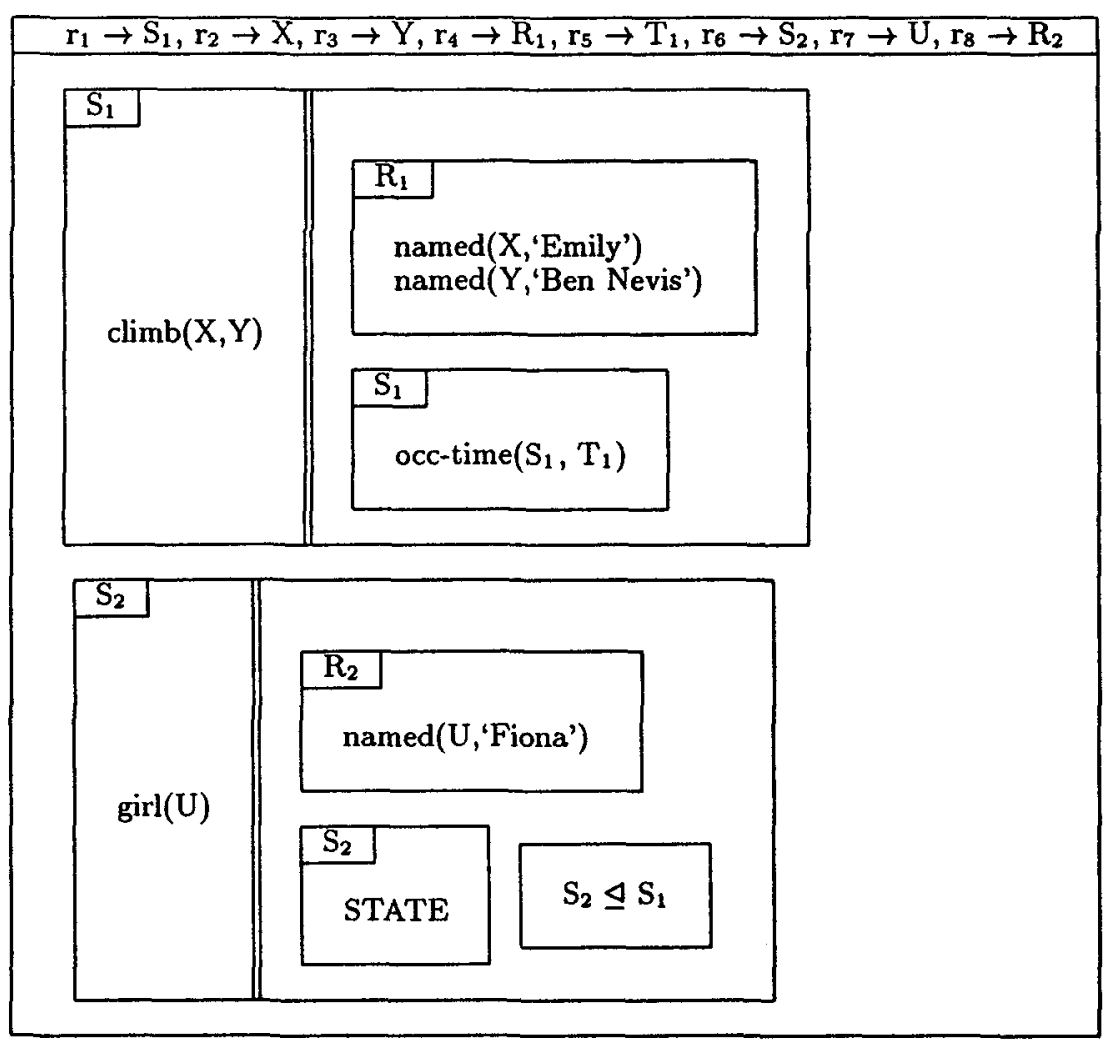

Figure 7: Representation for $(1 c, 1 e)$.

Finally, let us consider (1a,1f):

(1a) Emily climbed Ben Nevis in July.

(1f) Fiona was climbing Snowdon then.

In this case, an ETR is present and the second sentence has progressive aspect. This means that the conditions for both ETR 'then' and part-of 'then' are met.Our grammar will thus cause two representations to be generated for (1a,lf), corresponding to the two readings that we identified in Section 1.

\section{General remarks}

\subsection{Further Developments}

The system parses sequences of any length, keeping track of all the discourse referents/roles introduced so far. Thus, as it stands at present, it will find a temporal referent for 'then', irrespective of how far back in the discourse that referent was introduced. It may be desirable to refine this in some way-for example, to disallow anaphoric reference to an ETR that is more than a certain "distance" back in the discourse. Also, the system at present finds only the most recently introduce temporal referent. This could easily be modified-for example, in order to allow it to produce a set of alternatives. However, it appears that we would need to take discourse structure into account here.

\subsection{Relation to other accounts of temporal reference}

It is important to consider how our analysis fits with other work on temporal reference in discourse, and how readily our treatment of 'then' could be incorporated into these accounts. Kamp and Reyle (forthcoming) present a DRT fragment which deals with temporal reference but does not include 'then'. In (Glasbey, forthcoming) and (Glasbey, 1992) we present a modification of Kamp and Reyle's fragment which incorporates our analysis of 'then'. We make the necessary distinction between what we call "explicit" and "inferred" times by allowing a temporal referent to be introduced only when an explicit temporal referent is present. If there is no ETR, only an event referent may be introduced. This enables us to produce the correct readings for 'then'. We consider the ST/DRT account given in the present paper to be preferable, however, in that situation theory allows us to express information about the utterance in a way that traditional DRT does not. This enables us to make precisely the distinction we need between whether or not a particular referent was phonologically realised in the utterance.

Lascarides and Asher (1991) present an account of temporal reference where discourse relations between eventualities are deduced by means of defeasible reasoning. Their account is expressed in a version of 
DRT and preliminary investigations suggest that it could be extended to include 'then' in a similar way to the Kamp and Reyle fragment.

\section{Conclusion}

We have developed a computational grammar which parses discourse consisting of sequences of simple sentences containing a range of tense and aspect constructions. In particular, it generates the required readings for sentence-final 'then'. We have also indicated how our analysis of 'then' could be incorporated into some existing DRT accounts of temporal reference. The system appears to be capable of various refinements involving more detailed theories of discourse structure, and as such may provide a basis for development of more extensive systems for discourse analysis.

\section{Acknowledgments}

I would like to thank Robin Cooper, Max Cresswell, Elisabet Engdahl, Martin Mellor and Marc Moens for helpful advice and comments on this work.

\section{References}

[Bach, 1986] Emmon Bach. The algebra of events. Linguistics and Philosophy, 9:5-16, 1986.

[Barwise, 1989] Jon Barwise. The Situation in Logic. CSLI, Stanford, California, 1989.

[Barwise and Cooper, forthcoming] Jon Barwise and Robin Cooper. Extended Kamp Notation: a graphical notation for situation theory. In $\mathrm{P}$. Aczel, D. Israel, Y. Katagiri and S. Peters (eds.) Situation Theory and its Applications, Vol. 3. CSLI, Stanford, California, 1993.

[Cooper, 1991] Robin Cooper. Three lectures on situation theoretic grammar. In Natural Language Processing. Proceedings of 2nd Advanced School in Artificial Intelligence, Guarda, Portugal, October 8-12, 1990. In series: Lecture Notes in Artificial Intelligence, Miguel Filgueiras (ed.). Springer Verlag, Berlin, London, 1991.

[Cooper, forthcoming] Robin Cooper. Generalized quantifiers and resource situations. In P. Aczel, D. Israel, Y. Katagiri and S. Peters (eds.) Situation Theory and its Applications, Vol. 3. CSLI, Stanford, California, 1993.

[Cooper, ms1] Robin Cooper. Introduction to Situation Semantics. Edinburgh University, Department of AI and Centre for Cognitive Science. In preparation.

[Cooper, ms2] Robin Cooper. Situation theoretic discourse representation theory. Centre for Cognitive Science and Human Communication Research Centre, Edinburgh University, 1992. In preparation.
[Glasbey, 1992] Sheila Glasbey. Sentence-final 'then': a formal analysis. Edinburgh Research Papers in Cognitive Science, Centre for Cogniuve Science, Edinburgh University, 1992.

[Glasbey, forthcoming] Sheila Glasbey. Events and times: the semantics of 'then'. To appear in a forthcoming issue of Natural Language Semantics, 1993.

[Glasbey, msl] Sheila Glasbey. Event Structure in Natural Language Discourse. PhD thesis, Edinburgh University. In preparation.

[Glasbey, ms2] Sheila Glasbey. A formal analysis of 'the X' and 'the same X' in discourse. Centre for Cognitive Science, Edinburgh University. In preparation.

[Hinrichs, 1986] Erhard Hinrichs. Temporal anaphora in discourses of English. Linguistics and Philosophy, 9:63-82, 1986.

[Johnson and Klein, 1986] Mark Johnson and Ewan Klein. Discourse, anaphora and parsing. In Proceedings of the 11th COLING, 669-675, 1986.

[Kamp and Reyle, forthcoming] Hans Kamp and Uwe Reyle. From Discourse to Logic. Kluwer Academic Publishers, Dordrecht, 1993.

[Krifka, 1991] Manfred Krifka. Thematic relations as links between nominal reference and temporal constitution. In Ivan Sag and Anna Sabolcsi (eds.), Lexical Matters, Chicago University Press, 1991.

[Lascarides and Asher, 1991] Alex Lascarides and Nicholas Asher. Discourse relations and commonsense entailment. In Hans Kamp (ed.), Default Logics for Linguistic Analysis, Dyana Deliverable R2.5B, 1991.

[Mann and Thompson, 1987] W.C. Mann and S.A. Thompson. Rhetorical Structure Theory: A theory of text organization. Technical Report RR/87/190, Information Sciences Institute, Marina del Rey, California, 1987.

[Moens, 1987] Marc Moens. Tense, Aspect and Temporal Reference. Unpublished Ph.D. thesis, Edinburgh University, 1987.

[Smith, 1991] Carlota Smith. The Parameter of Aspect. Kluwer Academic Publishers, Dordrecht, 1991.

[Vendler, 1967] Zeno Vendler. Verbs and times. In Linguistics in Philosophy, Chapter 4, pages 97121. Cornell University Press, Ithaca, NY, 1967.

[Verkuyl, 1989] Henk Verkuyl. Aspectual classes and aspectual composition. Linguistics and Philosophy, 12:39-94, 1989.

[Vlach, 1981] Frank Vlach. The semantics of the progressive. In P. Tedeschi and A. Zaenen (eds.), Syntax and Semantics, Vol.14: Tense and Aspect. Academic Press, New York, 1981. 